\title{
VOTING DAPP IN EMBEDDED DEVICES USING BLOCKCHAIN TECHNOLOGY AND SECURITY CHALLENGES
}

\author{
Cristian TOMA \\ Department of Economic Informatics \& Cybernetics \\ Bucharest University of Economic Studies, Romania \\ cristian.toma@ie.ase.ro \\ Marius POPA \\ Department of Economic Informatics \& Cybernetics \\ Bucharest University of Economic Studies, Romania \\ marius.popa@ie.ase.ro \\ Mihai DOINEA \\ Department of Economic Informatics \& Cybernetics \\ Bucharest University of Economic Studies, Romania \\ mihai.doinea@ie.ase.ro \\ Bogdan IANCU \\ Department of Economic Informatics \& Cybernetics \\ Bucharest University of Economic Studies, Romania \\ bogdan.iancu@ie.ase.ro
}

\begin{abstract}
The paper presents the implementation challenges of a proof of concept development for a voting distributed application in an embedded device using the block-chain technology. First section is dedicated for the existing D-App voting solutions in the market and types of block-chains used for such applications. The second section shows the Ethereum (ETH) blockchain technical details that are used by the proof of the concept. In the third section, there are highlighted the implementation details for the voting D-App deployed into an embedded device. The last section shows the conclusions and the security challenges.
\end{abstract}

Keywords: Blockchain, DApp, Smart Contracts, embedded device, cybersecurity.

JEL classification: C88, L86, Y80

DOI: $10.12948 / \mathrm{ie} 2019.06 .11$

\section{Introduction and existing Voting DApp(s)}

Although multiple studies regarding the voting systems are done [6],[7], and multiple resources exist on the Internet about how to develop an e-voting application using block chain technology [8], [9], [10], [11], in the field, there are not many real implementations - especially in the embedded / IoT - Internet of Things area. The existing applications are not using the embedded devices and they are implemented directly into software on laptops/PC and only few of them with runnable code on smart phones. The proof of the concept presented in this paper highlights the security and advantage of the blockchain technology used into a e-voting application and launched a proposal of the improvement by using Java Card for the blockchain HD wallet and the smart phone device for the blockchain node which will run the application and the communications modules with other nodes from the proposed solution.

\section{Ethereum and Bitcoin Blockchain Technology}

Usually, the term Blockchain Technology refers to a collection of financial assets which allow to transfer the ownership of units of value securely. That collection of assets has specific 
characteristics like transparency, mistrust and public accessibility by using public key encryption schemes and proof of work methods.

Blockchain Technology eliminates the centralized control by a certain organization like a bank, company or government. It uses the decentralized consensus in order to keep the network functional. A better network security is achieved as much as the network grows and becomes more decentralized.

Briefly, a blockchain is a chain of chronological blocks. A block represents an aggregated set of data that is collected and processed by the so called process of mining. The aggregated set of data must fit inside the block. Each block is identified by a cryptographic hash and a timestamp. A new block contains a cryptographic hash of the previous block so that the blocks to make a chronologically ordered chain ever generated in the entire blockchain starting with the so called Genesis Block.

In the case of Bitcoin blockchain, a block contains data, Block Header, Block Identifies and Merkle Trees.

Block Header contains metadata for a particular block such as cryptographic hash of the previous block in the chain, mining competition and Merkle tree root to summarize the transactions within the block.

Block Identifies refers to the cryptographic hash that identifies a particular block uniquely.

Merkle Trees refer to the structure of transactions within the block. They are used to validate the content of big data structures. The root of the Merkle tree summarizes data in the related transactions and it is stored in the Block Header section.

Encryption is a core component of the Blockchain Technology. The asymmetric cryptography is used to ensure security for a blockchain-based network. In the asymmetric cryptography schemes, a key pair is used to perform encrypt/decrypt operations. The key used to encrypt the data is different from the key used to decrypt the data.

Public key cryptography offers mechanisms for key establishment (protocols to setup the keys over an insecure channel), digital signatures, identification, encryption and decryption.

There are three main classes of asymmetric algorithms [1], [4]:

- Integer factorization - large integers are used to perform asymmetric cryptography operations. RSA is the main cryptography scheme included in this category.

- Discrete logarithm - modular arithmetic is used to perform asymmetric cryptography operations. The modulo function is a one-way function so it is very difficult to find the input starting from the result. Diffie-Hellman is the common example for this kind of asymmetric cryptography.

- Elliptic curves - it is based on discrete logarithm but in the context of elliptic curves. Elliptic curve is an algebraic cubic curve over a field defined by an equation. The curve is non-singular and this is the main advantage of this kind of asymmetric algorithm. Also, elliptic curve cryptography needs small key size in comparison with RSA for the same security level. Examples based on elliptic curves are Elliptic Curve Digital Signatures Algorithm (ECDSA) and Elliptic Curve Diffie-Hellman (ECDH) key exchange.

Further, this paper will focus on Elliptic Curve Cryptography because the most known blockchain-based systems are based on that kind of cryptography scheme.

Elliptic curve is based on an equation that generates a curve over a finite field. Defined as an equation, the elliptic curve is:

$$
y^{2}=x^{3}+A x+B \bmod p
$$

A and B are parts of a finite field (Zp) or prime finite field (FP), and $\mathrm{p}$ is called point. 
The figure 1 highlights the elliptic curve as a real number representation, according to the equation over real numbers:

$$
y^{2}=x^{3}+A x+B
$$

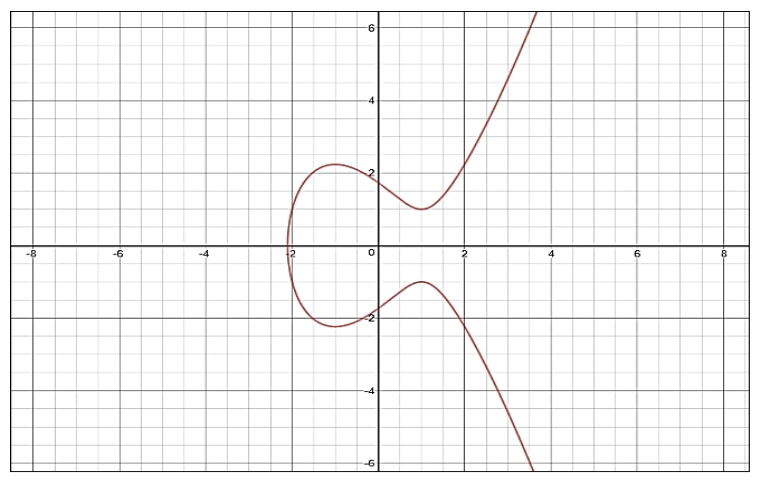

Figure 1. Elliptic curve over real numbers [4]

Over the previous elliptic curve graphical representation, a line could be drawn intersecting the elliptic curve in two points: $\mathrm{P}$ and $\mathrm{Q}$, figure 2 .

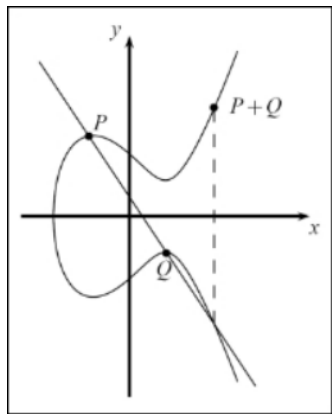

Figure 2. Add a point over real numbers [1]

The result of addition $\mathrm{P}+\mathrm{Q}$ is the third point called $\mathrm{R}$ on the elliptic curve. The $\mathrm{P}$ and $\mathrm{Q}$ real number coordinates are added according to the following equation:

$$
\left(x_{1}, y_{1}\right)+\left(x_{2}, y_{2}\right)=\left(x_{3}, y_{3}\right)
$$

where $\mathrm{P}$ has the coordinates $\left(\mathrm{x}_{1}, \mathrm{y}_{1}\right)$ and $\mathrm{Q}$ has the coordinates $\left(\mathrm{x}_{2}, \mathrm{y}_{2}\right)$.

The equation of point addition is:

$$
\begin{aligned}
& x_{3}=s^{2}-x_{1}-x_{2} \bmod p \\
& y_{3}=s\left(x_{1}-x_{3}\right)-y_{1} \bmod p
\end{aligned}
$$

As result:

$$
S=\frac{\left(y_{2}-y_{1}\right)}{\left(x_{2}-x_{1}\right)} \bmod p
$$

where $\mathrm{S}$ represents the line through the points $\mathrm{P}$ and $\mathrm{Q}$.

Point doubling is the process where $\mathrm{P}$ is added to itself. Figure 3 depicts how the line is drawn for point doubling. 


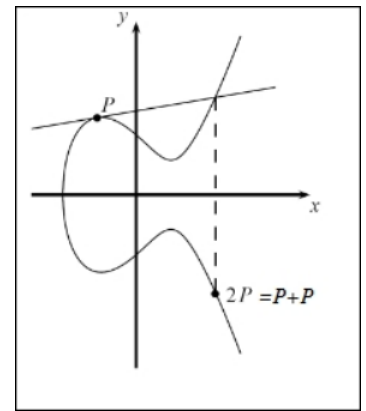

Figure 3. Point doubling over real numbers [1]

The equation of point doubling is:

where $\mathrm{P}$ has the coordinates $\left(\mathrm{x}_{1}, \mathrm{y}_{1}\right)$.

$$
\begin{aligned}
& x_{2}=s^{2}-2 x_{1} \bmod p \\
& y_{2}=s\left(x_{1}-x_{2}\right)-y_{1} \bmod p
\end{aligned}
$$

As result:

$$
S=\frac{3 x_{1}^{2}+a}{2 y_{1}} \bmod p
$$

where $\mathrm{S}$ is the slope of tangent line going through $\mathrm{P}$.

Under certain conditions, all points on an elliptic curve form a cyclic group. On an elliptic curve, the public key is a random multiple of the generator point. The private key is a randomly chosen integer used to generate the multiple. So, the public key is a point on elliptic curve, and the private key is a randomly chosen integer.

For instance, Bitcoin uses SECP256K1 specifications recommended by [5], section 2.4.1, figure 4.

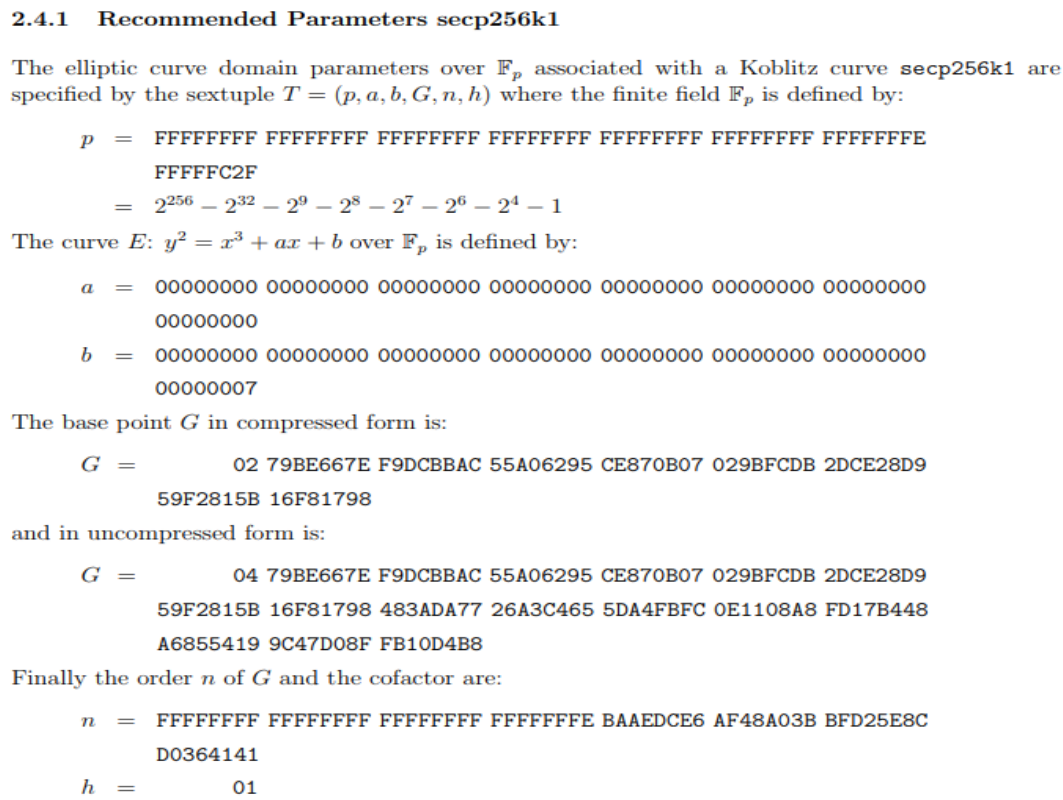

Figure 4. Recommended parameters for Elliptic Curve Cryptography used in Bitcoin [5]

The recommended SECP256K1 parameters have the following meanings:

- $\quad \mathbf{P}$ is the prime $\mathbf{p}$ and represents the size of the finite field. 
- $\quad \mathbf{a}$ and $\mathbf{b}$ are the coefficients of the elliptic curve equation.

- $\mathbf{G}$ is the base point that generates the required subgroup. It is called generator and could be represented both in compressed and un-compressed form.

- $\mathbf{n}$ is the order of the subgroup.

- $\quad \mathbf{h}$ is the cofactor of the subgroup.

The elliptic curve cryptography is used to generate Bitcoin address. A Bitcoin address is an identifier containing alphanumeric characters that represents a destination recipient for a Bitcoin transfer. Currently, there are three Bitcoin address formats: P2PKH, P2SH and Bech32. The generation of Bitcoin address is presented in figure 5 .

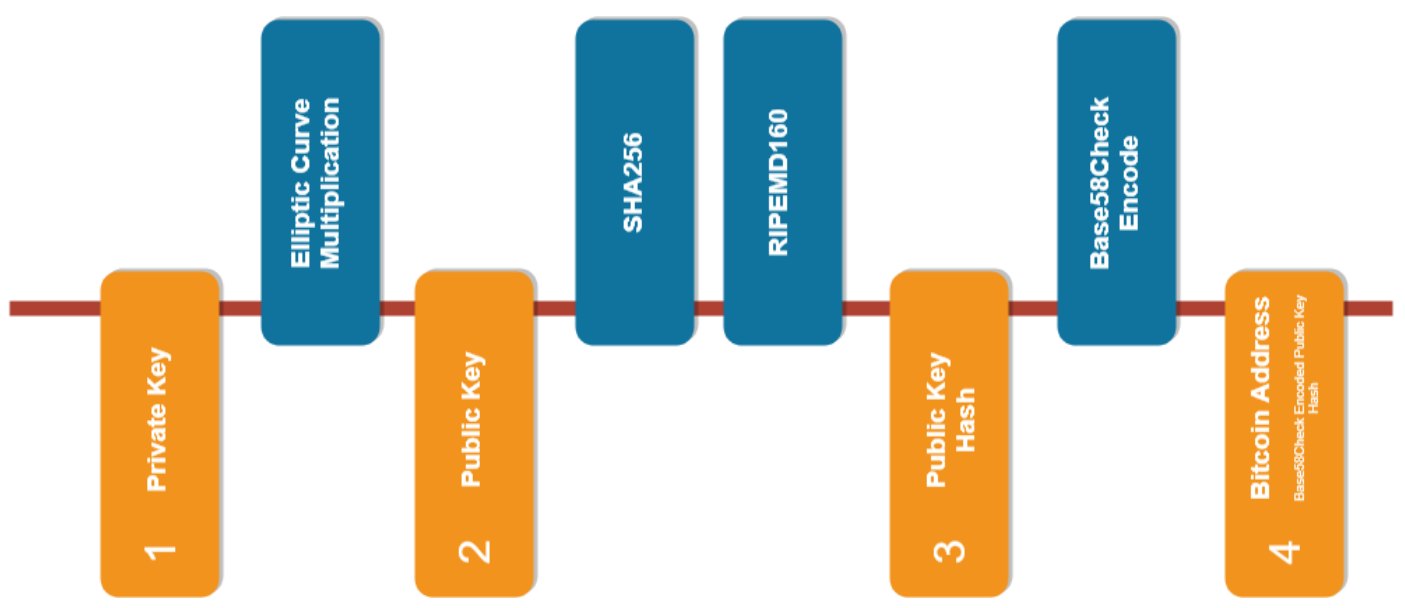

Figure 5. Generation of Bitcoin Address

Besides the Bitcoin address generation, the elliptic curve cryptography is used in blockchain to validate the integrity of the blocks chronologically.

Generation of the digital signature follows the function:

$$
\text { Sign }=F_{\text {sig }}\left(F_{\text {hash }}(t), p K e y\right) \text {, where: }
$$

- pKey is the signing elliptic curve private key.

- $t$ is the transaction or part of it.

- Fhash is the hashing algorithm.

- Fsig is the signing algorithm.

- Sign is the resulting signature.

As a data structure, a blockchain is a back-linked list of blocks of ordered transactions. Each block is identified by a hash value (SHA256 cryptographic hash algorithm) saved into the block header. According to Bitcoin implementation, the block structure contains several sections according to the following table.

Table 1. Bitcoin Block Structure

\begin{tabular}{|l|c|l|}
\hline \multicolumn{1}{|c|}{ Section name } & Section Length (Bytes) & \multicolumn{1}{c|}{ Description } \\
\hline Bloc Size & 4 & The size of a block in bytes \\
\hline Block Header & 80 & $\begin{array}{l}\text { Content of the Block Header structured } \\
\text { in fields }\end{array}$ \\
\hline Transaction Counter & 1 to 9 & Number of transactions \\
\hline Transactions & Variable & Records regarding the transaction data \\
\hline
\end{tabular}


www.conferenceie.ase.ro

The metadata about the block are stored within the Block Header. The structure of the Block Headers is detailed in table 2.

Table 2. Block Header Structure

\begin{tabular}{|l|c|l|}
\hline \multicolumn{1}{|c|}{ Section name } & Section Length (Bytes) & \multicolumn{1}{c|}{ Description } \\
\hline Version & 4 & Version number for tracking \\
\hline Previous Block Hash & 32 & $\begin{array}{l}\text { Hash value of the previous block in the } \\
\text { chain }\end{array}$ \\
\hline Merkle Root & 32 & $\begin{array}{l}\text { Hash value of the root for Merkle tree } \\
\text { regarding the current block transactions }\end{array}$ \\
\hline Timestamp & 4 & $\begin{array}{l}\text { Value regarding the time when the } \\
\text { block has been created }\end{array}$ \\
\hline Difficulty Target & 4 & $\begin{array}{l}\text { Difficulty target of Proof-of-Work } \\
\text { algorithm }\end{array}$ \\
\hline Nonce & 4 & Counter for Proof-of-Work algorithm \\
\hline
\end{tabular}

When a Bitcoin transaction is created, following actions must be performed:

- Hashing of the previous transaction.

- Hashing of the receiver's public key.

- Signing the transaction by using the sender's private key.

- Verification of the sender's signature.

Private keys, public keys or Bitcoin addresses could be stored by wallet software. Also, the wallet software could have additional functions to manage and carry out transactions. There are more wallet types [1], [2]:

- Non-deterministic wallets contain and manage private keys only. Challenges aim the management of a large number of keys and regular backups to prevent theft or loss.

- Deterministic wallets contain keys derived out of a seed value by using hash functions. The private key management is easier because the seed number could be used to recover the keys.

- Hierarchical deterministic wallets store keys in a tree structure derived from a seed. A master key is generated from the seed and derived keys are generated successively from the master key. The tree structure of keys is easily recoverable, maintainable and portable if the master key is known.

- Brain wallets store keys derived from a master key which is generated from the hash of passwords that are memorized. The attacks are possible by guessing or brute force methods.

- Paper wallets are paper-based wallets where the keys is printed on the physical material. The challenge is to ensure the physical security of the paper.

- Hardware wallets store keys on temper-resistant devices like secure element in NFCenabled phones.

- Online wallets store keys online and the access to them is made via cloud infrastructure services. The challenge is about the user trust in the online wallet service provider.

- Mobile wallets are installed on mobile devices. Such wallets use hardware capabilities of the mobile device to easily make transactions.

Besides the cryptocurrency transactions, the Blockchain Technology has become the base for Blockchain platform development by companies in order to get a safer and more effective business. 


\section{Voting DApp Solution implementation in Embedded Device}

Figure 6 shows the hardware architecture of the e-Voting D-App using Etherum blockchain technology connected to a Raspberry Pi board via GPIO pins..

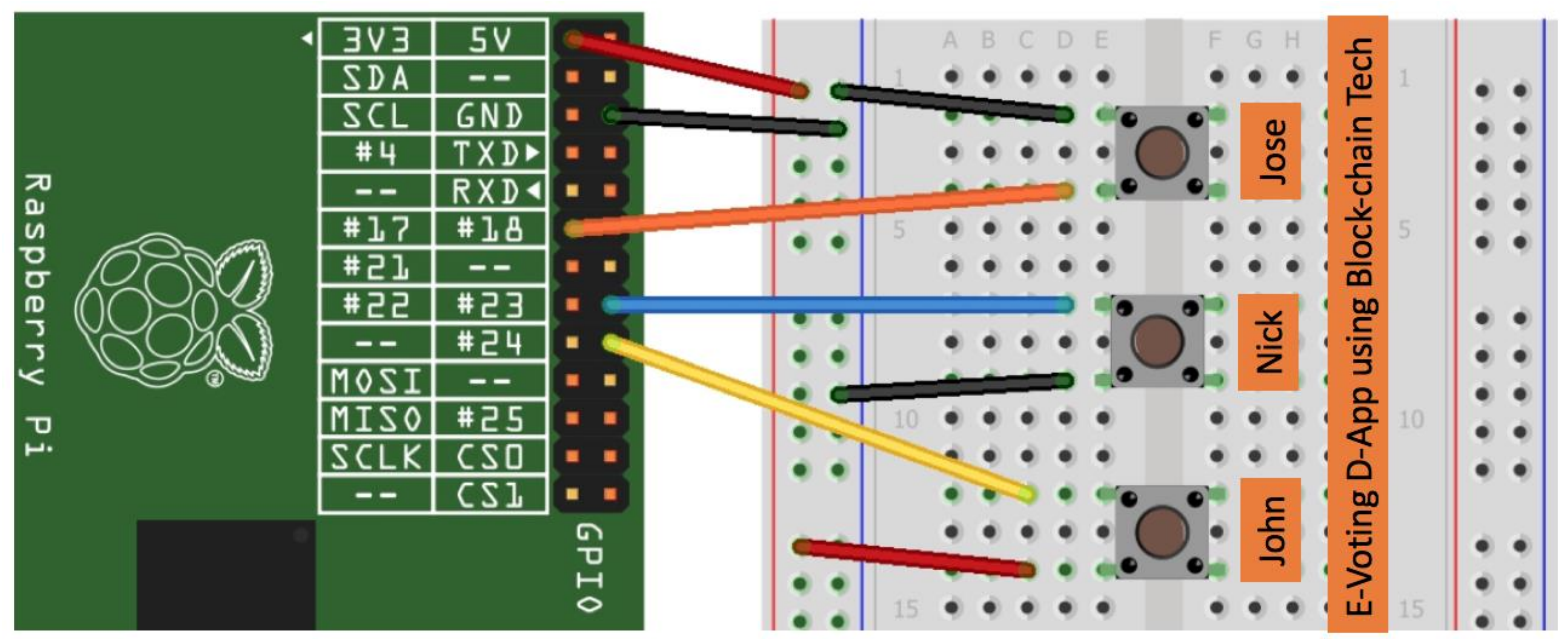

Figure 6. Hardware architecture of the e-Voting D-App using Etherum blockchain technology

The software components architecture is in figure 7 and the diagram shows all the involved components in a single node of this application (each voter has a board for the elections and the Ethereum node software running).

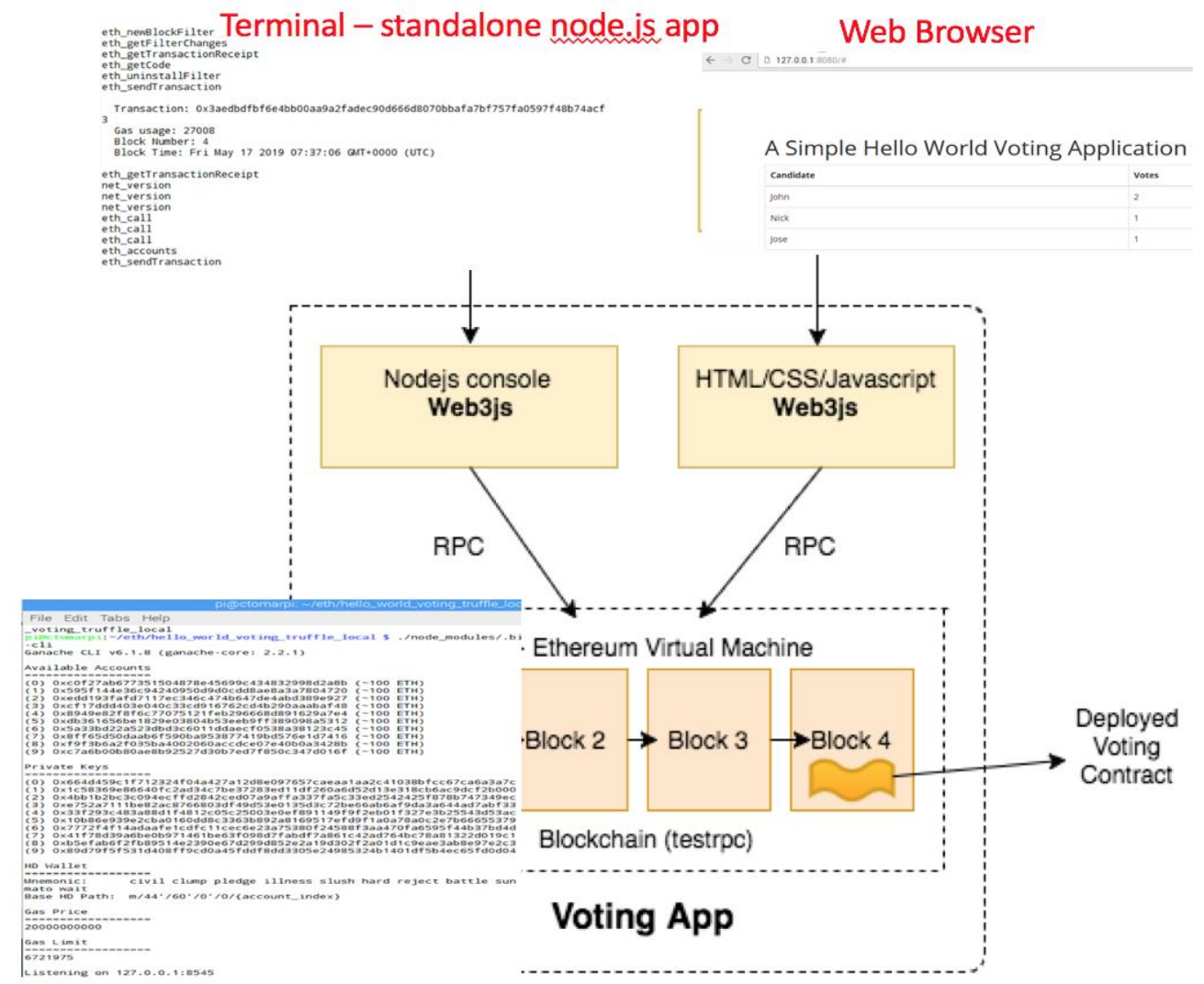

Figure 7. Software components within a node from the Ethereum blockchain 
In figure 7 from top left there is a terminal application in the embedded device which is running and interpreted in node.js. The buttons event from figure 6 are captured in Java DIO library over GPIO pin and then, a ECMAScript/JavaScript/node.js script is triggered to create RPC Remote Procedure Calls to the Ethereum Virtual Machine - EVM. The EVM is linked to an Ethereum wallet as MetaMask and the EVM is capable to run the smart contracts byte-code generated by solc compiler. Each vote is a transaction which is stored in the local node in the embedded device and then, the entire blockchain is synchronized with all the nodes within the network. Each of the voters has an embedded device for the election process. Figure 8 shows the fact that any of the voters can check in any moment the anonymous voting process and how many votes have each candidate in the election process.

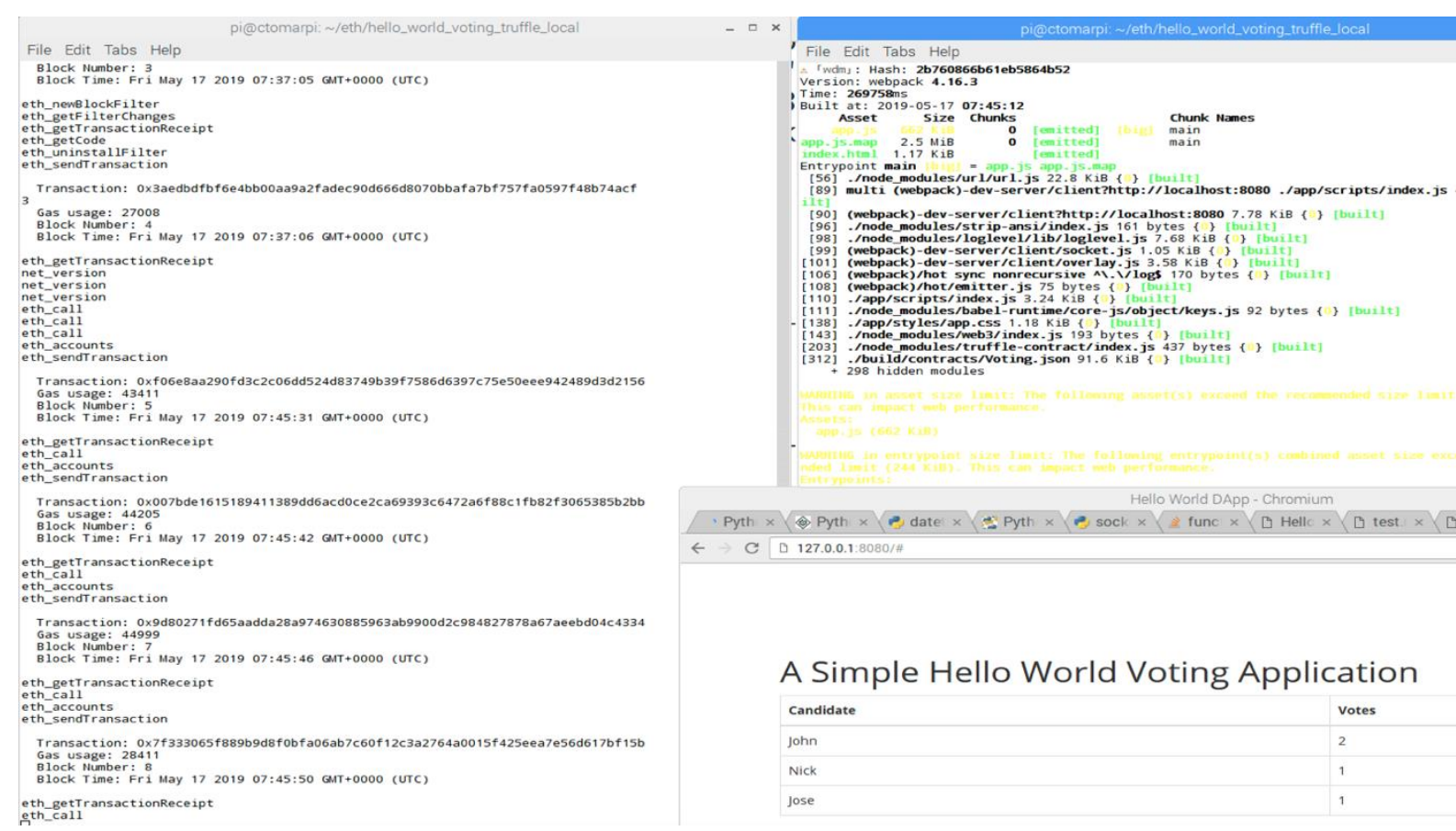

Figure 8. The web interface for displaying the votes number on each node

The top right of the figure 7 (the web browser results) are detailed in figure 8. For each registered vote a transaction is triggered in the global blockchain. From each wallet and node associated to a voter only one vote/one transaction with success can be made. In the end of the process all the nodes can see in a decentralized manner the votes numbers for each candidate.

\section{Conclusions and Security Challenges}

In terms of the security, each transaction is digitally signed and hashed with the cryptographic algorithms according with the second section of this paper - using Ethereum standards (e.g. for hash crypto function is usedSHA-3 with 256 bits block). The cryptographic algorithms are running on the CPU of the embedded device and for the moment, even if the controller is ARM, the application is not running in TEE - Trusted Execution Environment / ARM TrustZone. As a future development, the team is targeting to develop a Java Card applet which will run into a dual interface smart-card (contact and contactless) and to migrate the embedded node's machine code into a mobile application Then, from the mobile device, the candidate is chosen from the GUI of the mobile application and the transaction of the vote will be digitally signed and hashed by the smart card which is a tamper resistant CC/FIPS EAL 4+ certified state of the art secure element. The communication between the mobile application and the smart card can be done contactless via NFC - Near Field Communication / RFID chip of the smart phone. 
www.conferenceie.ase.ro

In the future phase, the proposed solution will be resilient to all known attacks from software, hardware, and logical point of view.

\section{Acknowledgment}

This paper presents results obtained within the PN-III-P1-1.2-PCCDI-2017-0272 ATLAS project ("Hub inovativ pentru tehnologii avansate de securitate cibernetică / Innovative Hub for Advanced Cyber Security Technologies "), financed by UEFISCDI through the PN III "Dezvoltarea sistemului national de cercetare-dezvoltare", PN-III-P1-1.2-PCCDI-2017-1 program.

\section{References}

[1] M. Antonopoulos. "Mastering Bitcoin, 2nd Edition", Publisher: O'Reilly Media, Inc., Release Date: June 2017, ISBN: 9781491954379.

[2] Andreas M. Antonopoulos, Gavin Wood, "Mastering Ethereum - Building Smart Contracts and Dapps", Publisher: O'Reilly Media, Inc., Release Date: 2018, https://covers.oreillystatic.com/images/0636920056072/rc_lrg.jpg.

[3] Konstantinos CHRISTIDIS, Michael DEVETSIKIOTIS, "Blockchains and Smart Contracts for the Internet of Things", IEEE Access, June 2016, Digital Object Identifier 10.1109/ACCESS.2016.2566339.

[4] Imran Bashir, "Mastering Blockchain - Master the theoretical and technical foundations of Blockchain technology and explore future of Blockchain technology", Packt Publishing, March 2017, ISBN 9781787125445

[5] SEC 2: Recommended Elliptic Curve Domain Parameters: http://www.secg.org/sec2v2.pdf

[6] Feng Hao, P.Y.A. Ryan and Piotr Zielinski. (2008). Anonymous voting by two-round public discussion. Available at: http://homepages.cs.ncl.ac.uk/feng.hao/files/OpenVote_IET.pdf

[7] Feng Hao and Piotr Zielinski. A 2-Round Anonymous Veto Protocol Available at: http://homepages.cs.ncl.ac.uk/feng.hao/files/av_net.pdf.

[8] Friðrik P. Hjálmarsson, Gunnlaugur K. Hreiðarsson, "Blockchain-Based E-Voting System", Online: https://skemman.is/bitstream/1946/31161/1/Research-Paper-BBEVS.pdf

[9] Timothy Ko, "A guide to developing an Ethereum decentralized voting application": https://medium.freecodecamp.org/developing-an-ethereum-decentralized-votingapplication-a99de24992d9

[10] Jackson Ng, "Voting on a Blockchain: DApp Demonstration": https://medium.com/coinmonks/voting-on-a-blockchain-dapp-demonstrationdfb5944a0c9e

[11] Mahesh Murthy, "Full Stack Hello World Voting Ethereum Dapp Tutorial": https://medium.com/@mvmurthy/full-stack-hello-world-voting-ethereum-dapp-tutorialpart-1-40d2d0d807c2 